1998

\title{
Surface smoothing and crystalline reorientation in thin cobalt films
}

\author{
H. T. Shi \\ D. Lederman
}

Follow this and additional works at: https://researchrepository.wvu.edu/faculty_publications

\section{Digital Commons Citation}

Shi, H. T. and Lederman, D., "Surface smoothing and crystalline reorientation in thin cobalt films" (1998). Faculty Scholarship. 360. https://researchrepository.wvu.edu/faculty_publications/360

This Article is brought to you for free and open access by The Research Repository @ WVU. It has been accepted for inclusion in Faculty Scholarship by an authorized administrator of The Research Repository@ WVU. For more information, please contact ian.harmon@mail.wvu.edu. 


\title{
Surface smoothing and crystalline reorientation in thin cobalt films
}

\author{
H. T. Shi and D. Lederman \\ Physics Department, West Virginia University, Morgantown, West Virginia 26506-6315 \\ (Received 5 March 1998; revised manuscript received 20 April 1998)
}

\begin{abstract}
Epitaxial cobalt films in the thickness range of 3.9 to $8.6 \mathrm{~nm}$ were deposited on $\mathrm{Al}_{2} \mathrm{O}_{3}[11 \overline{2} 0]$ substrates by dc magnetron sputtering at a substrate temperature of $315^{\circ} \mathrm{C}$. In situ annealing was performed in a vacuum after which the samples were rapidly quenched to room temperature in order to preserve the high temperature structure. Ex situ atomic-force microscopy revealed that surface roughening takes place during annealing and reaches a maximum when the annealing temperature $T_{A}$ is equal to a critical temperature $T_{C} \sim 500{ }^{\circ} \mathrm{C}$. We discovered that if $T_{A}>T_{C}$ the surface becomes smooth again, although large rectangular pits that go down to the substrate also appear. X-ray-diffraction data show that unannealed samples are oriented along the hcp[0001] direction. Upon annealing samples transform to a preferentially fcc[111] orientation for $T_{A}<T_{C}$, and subsequently to a fcc[001] orientation for $T_{A}>T_{C}$. We show that surface or interface oxidation cannot be the sole cause of this effect. We speculate that an increasing interface strain at higher temperatures or a surface reconstruction of the substrate are possible mechanisms. [S0163-1829(98)50128-2]
\end{abstract}

There is much interest in the study of Co thin films because of the ease of epitaxial growth on several types of substrates its uniaxial magnetic anisotropy at room temperature, and its phase transformation from a high-temperature face-centered-cubic (fcc) structure to a hexagonal closedpacked (hcp) structure at a temperature $\sim 415{ }^{\circ} \mathrm{C}$. ${ }^{1}$ A significant portion of the Co film research has focused on the dependence of the crystallinity, surface characteristics, and magnetic properties on the growth temperature on different types of substrates including monocrystalline $\mathrm{Al}_{2} \mathrm{O}_{3},{ }^{2-5}$ $\mathrm{MgO},{ }^{6}$ and $\mathrm{Cu}^{7,8}$ These studies have revealed that $\mathrm{Co}$ films thicker than $10 \mathrm{~nm}$ deposited on $\mathrm{Al}_{2} \mathrm{O}_{3}[11 \overline{2} 0]$ at approximately $300{ }^{\circ} \mathrm{C}$ are smoother than if grown at higher temperatures. Similar surface roughening occurs in a number of different systems including semiconductors, ${ }^{9,10} \mathrm{Ag}[115],{ }^{11}$ $\mathrm{Ag}[111],^{12}$ and $\mathrm{Ag}[100] .^{12}$ Other groups ${ }^{13-16}$ have investigated the phase stability and phase transformation of Co fine particles, where the fcc phase is stable only at high temperatures. While the origin of this behavior in Co particles remains ambiguous, few studies have been performed on the role of the thermal treatment processes for Co films in the thickness range of $10 \mathrm{~nm}$.

Here we report on the effect of thermal annealing on the surface roughness and crystallinity of epitaxial Co thin films grown on $\mathrm{Al}_{2} \mathrm{O}_{3}[11 \overline{2} 0]$. Ex situ atomic-force microscopy (AFM) and x-ray diffraction (XRD) were employed to characterize the surface roughness and structural evolution. The samples were quenched to room temperature after annealing to preserve the high temperature metastable phase. For annealing temperatures $T_{A}<T_{C}$ where $T_{C} \sim 500{ }^{\circ} \mathrm{C}$ is a critical temperature, the surface roughness increases with $T_{A}$, in agreement with previous work. However, we discovered that if $T_{A}>T_{C}$ the surface becomes smooth once again, although large rectangular pits are formed which reach all the way down to the substrate. The smoothing of the surface coincides with a crystalline reorientation of the film from fcc[111] to fcc[001].

All samples were deposited on $\mathrm{Al}_{2} \mathrm{O}_{3}[11 \overline{2} 0]$ substrates by dc magnetron sputtering in a high vacuum chamber at a base pressure of $4 \times 10^{-7}$ Torr. The Ar partial pressure was 3.0 mTorr. A $99.9 \%$ pure Co target was mounted approximately $10 \mathrm{~cm}$ below the substrate holder. Up to five substrates were mounted on a rotatable stainless steel plate. Each substrate was independently heated by placing it under a heater above the sputtering gun. The growth rate, nominally $0.04 \mathrm{~nm} / \mathrm{s}$, was controlled by a calibrated quartz crystal monitor. Substrates were degreased in trichloroethylene acetone, and methanol, and then etched in a mixture of sulfuric and phosphoric acids before being loaded in the chamber. The substrate surface temperature was determined by bonding a 0.5 mm diameter thermocouple thermometer to an $\mathrm{Al}_{2} \mathrm{O}_{3}$ substrate using a graphite compound. The heater's temperature sensor was calibrated against the thermocouple bonded to the substrate. All of the temperatures referred to here correspond to the actual surface temperature. Before growth substrates were heat cleaned at $566^{\circ} \mathrm{C}$ in vacuum for $15 \mathrm{~min}$. The growth temperature for all the samples was $315^{\circ} \mathrm{C}$. In situ annealing was carried out in vacuum at a pressure $<1.5$ $\times 10^{-6}$ Torr, immediately after growth for $15 \mathrm{~min}$. Annealed samples were rapidly quenched to room temperature by turning off the heater and rotating the sample holder away from the heater. The root-mean-square (RMS) surface roughness was determined from AFM images performed in air at room temperature. The diameter of the $\mathrm{AFM} \mathrm{SiN}_{3}$ tip was $\sim 40 \mathrm{~nm}$. Because the lateral size of the islands formed at higher temperatures were on the order of $\sim 300 \mathrm{~nm}$ or larger and the scan areas were $3000 \mathrm{~nm} \times 3000 \mathrm{~nm}$, the AFM RMS roughness represents an accurate measure of the surface roughness at the length scales of interest which are much larger than the tip diameter. XRD was performed using a 18 $\mathrm{kW}$ rotating anode $\mathrm{x}$-ray generator with a four-circle diffractometer using $\mathrm{Cu} K_{\alpha}(\lambda=0.15418 \mathrm{~nm})$ radiation.

Figure 1 shows the AFM images for samples with a thickness of $6.3 \mathrm{~nm}$. The sample in Fig. 1(a) was not annealed while those in $1(\mathrm{~b})$ and 1 (c) were annealed at $503{ }^{\circ} \mathrm{C}$ and $566{ }^{\circ} \mathrm{C}$, respectively. Note that the surface of the sample in Fig. 1(b) is rougher than the surface of the other two images, 

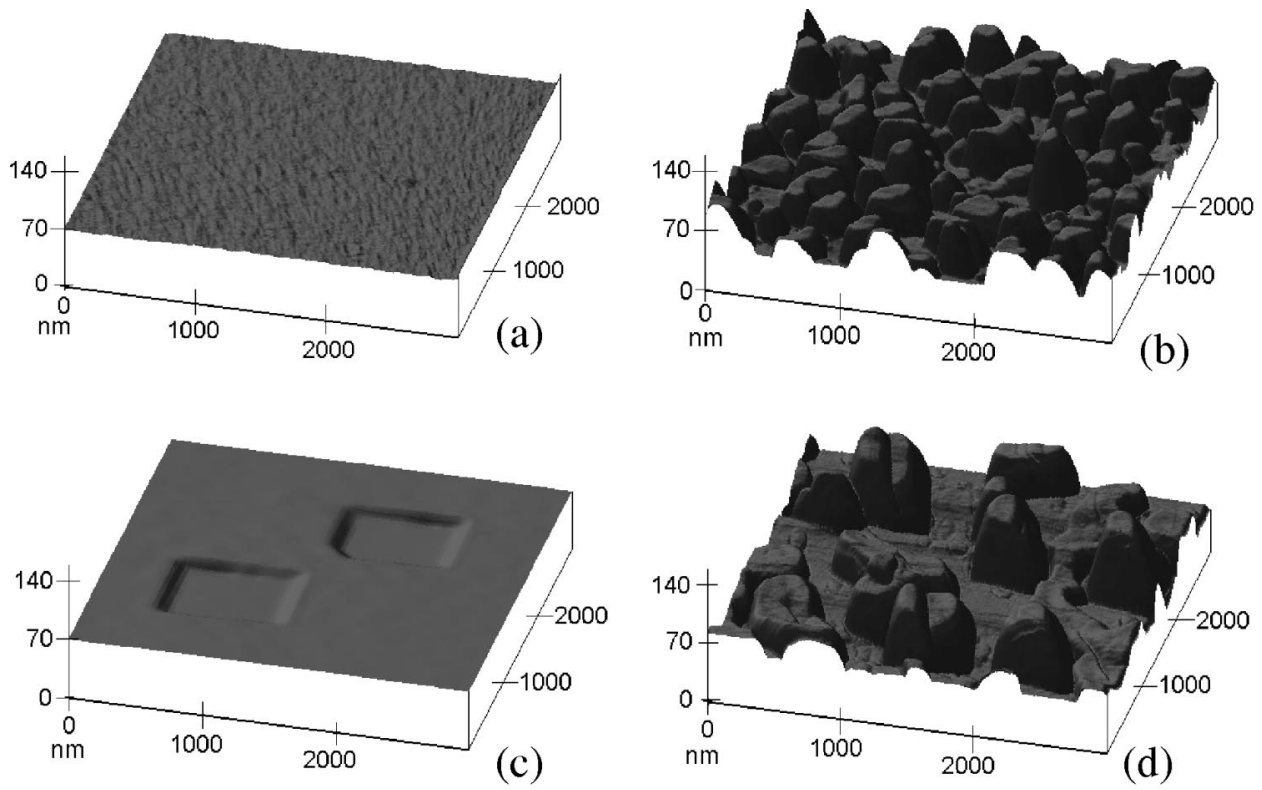

FIG. 1. AFM images of Co films $6.3 \mathrm{~nm}$ in thickness when grown at $315^{\circ} \mathrm{C}$. (a) is the surface of the as-grown sample (b) was annealed at $503{ }^{\circ} \mathrm{C}$, and (c) was annealed at $565^{\circ} \mathrm{C}$. Samples in (b) and (c) were quenched to room temperature after annealing. Image (d) corresponds to a sample that was annealed at $566^{\circ} \mathrm{C}$ and then cooled at $20^{\circ} \mathrm{C} / \mathrm{min}$ to $100{ }^{\circ} \mathrm{C}$.

and that the surface of the sample annealed at $566^{\circ} \mathrm{C}$ is very smooth except for the rectangular pits evident in the image. Clearly annealing at higher temperatures does not always result in a rougher surface.

In order to examine the dependence of the surface roughness on the annealing temperature for different film thicknesses we deposited two other series of samples with nominal thicknesses of 3.9 and $8.6 \mathrm{~nm}$. As shown in Fig. 2(a) samples with different thicknesses have similar temperaturedependent surface features with $T_{C} \sim 500{ }^{\circ} \mathrm{C}$ for the three thicknesses. The main difference is that thinner films are in general smoother than thicker films for $T_{A}<T_{C}$.

A complete understanding of these surface roughening and smoothing transitions requires a thorough knowledge of the crystalline structure which we studied via XRD. The XRD measurements were carried out using three types of scans: (1) out-of-plane scans with the x-ray wave vector $\mathbf{q}$ parallel to the growth direction; (2) off-specular scans, with q having a component parallel to the surface; and (3) $\phi$ scans, with q kept fixed while the sample is rotated about the growth direction.

Typical out-of-plane scans are shown in the inset of Fig. 2(b). The peaks at $2 \theta=37.78^{\circ}$ correspond to the $\mathrm{Al}_{2} \mathrm{O}_{3}(11 \overline{2} 0)$ reflection; peaks at $2 \theta \approx 44.4^{\circ}$ correspond to the hcp(0002) or fcc(111) reflections; and peaks at $2 \theta$ $=51.8^{\circ}$ correspond to the fcc $(002)$ reflection. As $T_{A}$ increases the fcc (002) reflection becomes stronger. In fact, for the sample annealed at $566{ }^{\circ} \mathrm{C}$, only the fcc $(002)$ reflection is visible indicating that the film has undergone a profound reorientational transition. This is discussed further below.

The full widths at half maximum (FWHM) of the $2 \theta$ $\approx 44.4^{\circ}$ peaks decrease as $T_{A}$ increases for $T_{A}<T_{C}$ and its intensity decreases rapidly for $T_{A}>T_{C}$. The decrease in the FWHM is related to the formation of the tall structures in Fig. 1(b), which are crystalline and therefore have a larger structural coherence along the growth direction than the unannealed film. This is illustrated more explicitly in Fig. 2(b), which shows the approximate structural coherence length $L_{C}$, calculated from the FWHM of the out-of-plane peak using Scherrer's equation, as a function of $T_{A} . L_{C}$ increases with $T_{A}$, reaching a maximum value at $T_{C} \sim 500{ }^{\circ} \mathrm{C}$. For $T_{A}>T_{C}$, the films become smooth again and $L_{C}$ decreases. We conclude that the islands become on the order of 32-nm tall for the 6.3- and 8.6-nm-thick samples at $T$ $\sim T_{C}$.

The inset in Fig. 2(b) provides additional evidence that the samples experience smooth-to-rough and then rough-tosmooth surface phase transitions. For the unannealed sample and the sample annealed at the highest temperature $\left(566{ }^{\circ} \mathrm{C}\right)$ finite-size peaks appear around the peak at hcp(0002) and fcc(002) peaks. Although the film annealed at $566^{\circ} \mathrm{C}$ does not have fringes as clear as those of the unannealed sample, they are nevertheless evident and confirm that the surface becomes smooth again at higher temperatures. In addition, a calculation of the film thickness based on the position of the interference fringes yields film thicknesses of $6.4 \mathrm{~nm}$ and 6.3 $\mathrm{nm}$ for the $\mathrm{hcp}(0002)$ and fcc(002) reflections, respectively, which are in good agreement with the nominal thickness of $6.3 \mathrm{~nm}$ and with the $6.4 \mathrm{~nm}$ average step height of the pits in Fig. 1(c).

Bulk Co undergoes a phonon-driven martensitic transformation from a low-temperature hcp to a high-temperature fcc phase with a transition temperature $\sim 415^{\circ} \mathrm{C} .{ }^{17}$ As-grown Co films usually exist in a combination of the hcp and fcc structures. ${ }^{2}$ Therefore the peak at $2 \theta \approx 44.4^{\circ}$ in principle represents a mixture of the $\mathrm{hcp}(0002)$ and $\mathrm{fcc}(111)$ reflections. In order to determine the relative contributions of these two phases, off-specular XRD scans of in-plane hcp(1013) and fcc(220) reflections were performed. The as-grown samples did not have any fcc(220) reflections, indicating that the unannealed samples are mostly hcp. However, as $T_{A}$ approaches $T_{C}$, the hcp and fcc structures begin to coexist. 


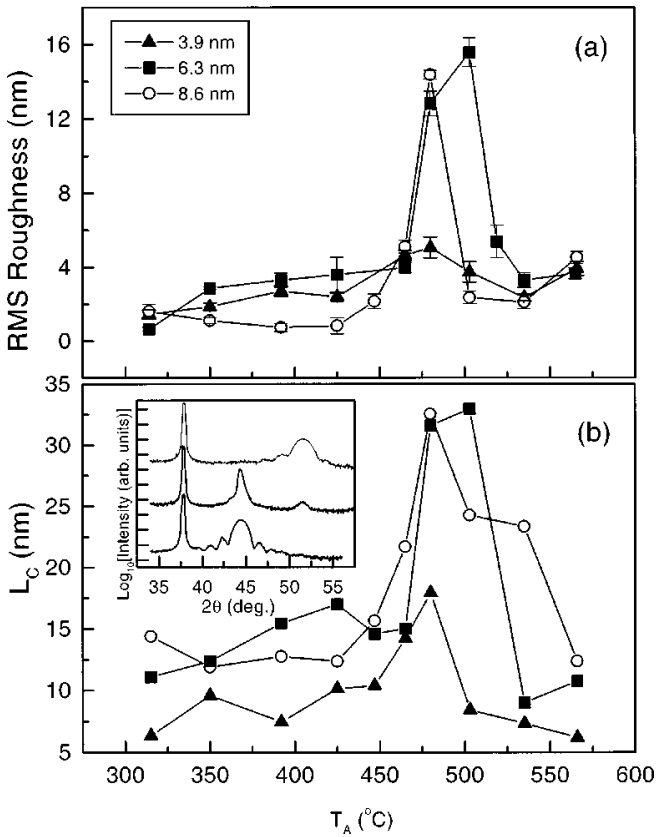

FIG. 2. (a) AFM RMS height fluctuations for Co films with different nominal thickness $t$ as a function of the annealing temperature $T_{A}$. The error bars represent the standard deviation of measurements of several images. (b) Structural coherence length $L_{C}$ along the growth direction determined from the width of the most intense peak using Scherrer's equation. Inset: Wide angle XRD scans of $t=6.3 \mathrm{~nm}$ samples. From bottom to top they correspond to the unannealed sample, the sample annealed at $503{ }^{\circ} \mathrm{C}$, and the sample annealed at $566{ }^{\circ} \mathrm{C}$. For clarity the scans have been displaced from each other. The peak at $2 \theta \sim 44.4^{\circ}$ corresponds to the hcp (0002) and/or fcc(111) reflections, while the peak at $2 \theta \sim 51.8^{\circ}$ corresponds to the fcc $(002)$ reflection. Additional peaks around these two peaks are due to the interference between the top and bottom surfaces of the film. The peak at $2 \theta=37.78^{\circ}$ is a substrate (11̄ㅇ) reflection.

More interestingly when $T_{A}>T_{C}$, the Co islands change their crystallographic orientation from fcc[111] to fcc[001]. This transformation coincides with temperature at which the surface becomes smooth again. Figure 3 shows how the fcc(002) peak area, which is proportional to the amount of the fcc[001] phase present in the samples, increases with annealing temperature. A rapid increase in the peak area occurs at $T_{A} \sim T_{C}$.

Figure 4 shows $\phi$ scans of the three samples shown in Figs. 1(a)-1(c) which shows the epitaxial relationship between the films and the substrate. The scan in Fig. 4(a) was performed at the Bragg condition for the hcp(1013) reflection, while the scan in (b) was performed at the fcc(220) Bragg condition. Both of these scans show the sixfold symmetry characteristic of hcp[0001] or fcc[111] epitaxial growth. From a similar scan of the $\mathrm{Al}_{2} \mathrm{O}_{3}(22 \overline{4} 6)$ peak, we determined that the orientation in the plane was Co hcp[11220] or fcc[101] $\| \mathrm{Al}_{2} \mathrm{O}_{3}[0001]$. The scan shown in 4(c) was performed at the fcc(113) Bragg condition for the fcc[001]-oriented film annealed at $566^{\circ} \mathrm{C}$. The evident fourfold symmetry is characteristic of a definite epitaxial relationship between the substrate and the film. In this case the epitaxial relation is Co fcc[110] $\| \mathrm{Al}_{2} \mathrm{O}_{3}$ [0001]. In addition, from in-plane and out-of-plane XRD measurements of the

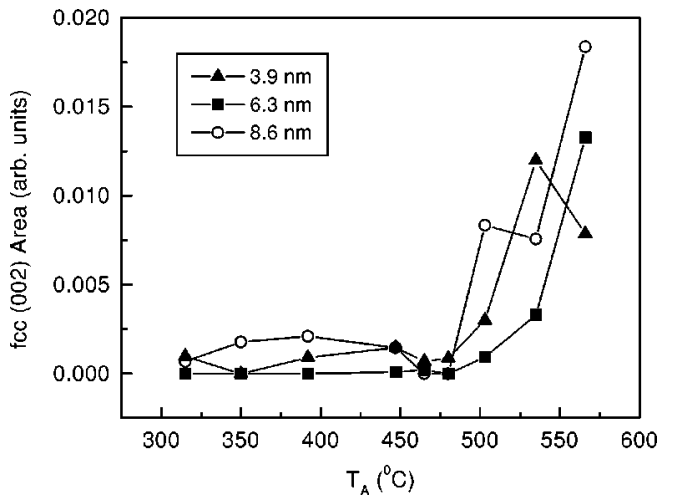

FIG. 3. The fcc(002) peak area as a function of the annealing temperature $T_{A}$. The solid lines are guides to the eye.

fcc(113) and fcc(002) peaks, we obtained an out-of-plane lattice parameter of $0.353 \mathrm{~nm}$ and an in-plane lattice parameter of $0.359 \mathrm{~nm}$. Because the bulk Co fcc lattice parameter is $0.354 \mathrm{~nm}$ the Co is strained in the plane and the out-ofplane lattice parameter probably decreases to accomodate this strain.

At lower temperatures there is only a slight difference in the free energies between the bulk Co hcp and fcc structures which is estimated to be ${ }^{18} E_{\mathrm{fcc}}-E_{\mathrm{hcp}}=0.06 \mathrm{kcal} / \mathrm{mol}$. It is this small energy difference together with phonon interactions that causes bulk Co to become fcc above $415^{\circ} \mathrm{C}$. Therefore, our as-grown samples usually are hcp[0001] oriented. Increasing the annealing temperature results in a high

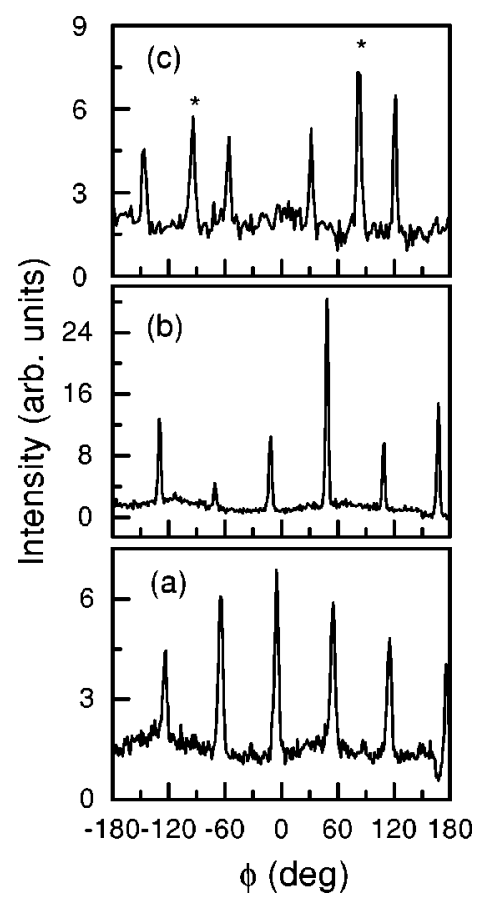

FIG. 4. $\phi$ scans of samples with an unannealed nominal thickness of $6.3 \mathrm{~nm}$. (a) is a scan of the hcp (1013) peak of the unannealed sample; (b) is a scan of the fcc(220) peak for the sample annealed at $503{ }^{\circ} \mathrm{C}$; and (c) is a scan of the fcc $(113)$ peak for a sample annealed at $566^{\circ} \mathrm{C}$. The peaks at $\phi=-95^{\circ}$ and $\phi=85^{\circ}$ in-

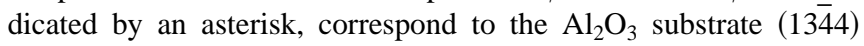
reflection. The film orientations are (a) hcp[0001], (b) fcc[111], and (c) fcc[001]. 
atomic mobility, and in order to relieve the epitaxial strain, the film forms large islands. Because the fcc phase is stable at higher temperatures, these islands are fcc[111] oriented.

The mechanism that causes the surface to become smooth for $T_{A}>T_{C}$ is unclear. One possibility is that oxidation could drive the smoothing transition. This could occur either at the film/vacuum interface due to a small oxygen or water partial pressure, or at the film/substrate interface, due to oxygen migration from the substrate. ${ }^{19}$ We investigated this possibility by cooling a sample annealed at $566^{\circ} \mathrm{C}$ at a rate of $20{ }^{\circ} \mathrm{C} /$ min to $100{ }^{\circ} \mathrm{C}$ instead of quenching. The AFM image of this sample is shown in Fig. 1(d). The surface morphology of this sample is reminiscent of the fcc[111]-oriented samples. Indeed, the XRD scan showed that the slowly cooled sample is a mixture of $\mathrm{fcc}[111]$ and $\mathrm{fcc}[001]$ orientations indicating that the smoothing transition in Co is reversible. If oxidation did play an important role in the smoothing transition, it is difficult to understand why the process would be reversible. In any case, oxidation should be more pronounced in the sample that is cooled slowly, which would make reversibility even more difficult. Although we do not claim that our films do not oxidize, we believe that it is unlikely that oxidation is responsible for the reorientation of the fcc surface.

Another possibility is that the different thermal expansion coefficients of the film and substrate change the strain as a function of temperature. The thermal expansion coefficient $\alpha$ of $\mathrm{Al}_{2} \mathrm{O}_{3}$ between $20{ }^{\circ} \mathrm{C}$ and $600{ }^{\circ} \mathrm{C}$ is $\alpha_{\perp} \sim 7.3 \times 10^{-6} \mathrm{~K}^{-1}$ and $\alpha_{\|} \sim 8.1 \times 10^{-6} \mathrm{~K}^{-1}$ perpendicular and parallel to the $c$ axis, respectively, ${ }^{20}$ while for bulk Co (Ref. 21) $\alpha \sim 14 \mathrm{~K}^{-1}$. At higher temperatures the interfacial strain increases because of the difference in thermal expansion coefficients until a temperature is reached at which the atomic mobility enables the film to reorient itself to relieve this strain. When the film is quenched, the high temperature phase is preserved.

Finally, it is also possible that the surface of the $\mathrm{Al}_{2} \mathrm{O}_{3}$ substrate undergoes a surface reconstruction at $T_{C}$ which favors the formation of fcc[001] Co. However, to our knowledge, the surface of $\mathrm{Al}_{2} \mathrm{O}_{3}[11 \overline{2} 0]$ does not reconstruct at $\sim 500{ }^{\circ} \mathrm{C}$. This means that the substrate surface reconstruction would have to occur because of an interaction between the Co and the substrate's surface.

In closing we note that a similar, as yet unexplained smoothing transition occurs in Ag fcc[111] films grown at $130 \mathrm{~K}$ on GaAs when they are annealed at room temperature. $^{22}$ In the case of the Ag films the smoothing transition occurs for films thicker than $1.5 \mathrm{~nm}$. As in our Co films, rectangular pits are also formed, although the $\mathrm{Ag}$ pit areas $\left(\sim 2.0 \times 2.0 \mathrm{~nm}^{2}\right)$ are smaller. This could indicate that the observed phenomena is intrinsic to fcc transition metal surfaces.

To summarize the surface of epitaxial Co films grown on $\mathrm{Al}_{2} \mathrm{O}_{3}$ at high temperatures was studied by annealing them at high temperatures and then quenching them to room temperature. We find that surface roughening occurs for $T_{A}<500{ }^{\circ} \mathrm{C}$ in agreement with previous investigations. However, we discovered that for $T_{A}>500{ }^{\circ} \mathrm{C}$ the surface becomes smooth once again with the formation of large rectangular pits. The smoothing transition is accompanied by a fcc[111] to fcc[001] reorientation of the film. We find that oxidation at the substrate or vacuum interfaces is an unlikely cause for this smoothing/reorientation transition. Increasing interfacial strain at high temperatures and a substrate surface reconstruction at the film/substrate interface are possible causes that require further investigation.

We are grateful to A. Manivannan for his initial help in obtaining AFM images and T. Charlton for helping with the growth and x-ray characterization at the initial stages of this project. We also thank T. Myers, J. Weaver, and Z. Zhang for fruitful discussions. This work was supported by the West Virginia NASA Space Grant Consortium.
${ }^{1}$ Constitution of Binary Alloys, edited by M. Hansen (McGrawHill, New York, 1958), p. 471ff.

${ }^{2}$ Ch. Morawe, A. Stierle, N. Metoki, K. Brohl, and H. Zabel, J. Magn. Magn. Mater. 102, 223 (1991).

${ }^{3}$ A. Stierle, A. Abromeit, N. Metoki, and H. Zabel, J. Appl. Phys. 73, 4808 (1993).

${ }^{4}$ K. Hara, K. Itoh, M. Kamiya, K. Okamoto, and T. Hashimoto, J. Magn. Magn. Mater. 161, 287 (1996).

${ }^{5}$ N. Metoki, T. Zeidler, A. Stierle, K. Brohl, and H. Zabel, J. Magn. Magn. Mater. 118, 57 (1993).

${ }^{6}$ M. Hashimoto, H. Qiu, T. Ohbuchi, M. Adamik, H. Nakai, A. Barna, and P. B. Barna, J. Cryst. Growth 166, 792 (1996).

${ }^{7}$ D. Pescia, G. Zampieri, M. Stampanoni, G. L. Bona, R. F. Willis, and F. Meier, Phys. Rev. Lett. 58, 933 (1987).

${ }^{8}$ H. Li and B. P. Tonner, Surf. Sci. 237, 141 (1990).

${ }^{9}$ U. Bangert, A. J. Harvey, C. Dieker, H. Hartdegen, L. Vescan, and A. Smith, J. Appl. Phys. 78, 811 (1995).

${ }^{10}$ C. S. Ozkan, W. D. Nix, and H. Gao, Appl. Phys. Lett. 70, 2247 (1997).

${ }^{11}$ M. S. Hoogeman, D. C. Schloßer, J. B. Sanders, L. Kuipers, and J. W. M. Frenken, Phys. Rev. B 53, 13299 (1996).
${ }^{12}$ W. C. Elliott, P. F. Miceli, T. Tse, and P. W. Stephens, Phys. Rev. B 54, 17938 (1997).

${ }^{13}$ Y. Fukano, J. Appl. Phys. 13, 1001 (1974).

${ }^{14}$ C. G. Granqvist and R. A. Buhrman, J. Appl. Phys. 47, 2200 (1976).

${ }^{15}$ J. P. Chen, C. M. Sorensen, K. J. Klabunde, and G. C. Hadjipanayis, J. Appl. Phys. 76, 6316 (1994).

${ }^{16}$ J. Jiao, S. Seraphin, X. Wang, and J. C. Withers, J. Appl. Phys. 80, 103 (1996).

${ }^{17}$ S. M. Shapiro and S. C. Moss, Phys. Rev. B 15, 2726 (1977).

${ }^{18}$ Handbook of Chemistry and Physics, 66th ed., edited by C. Weast (CRC, Boca Raton, FL, 1987), p. D-43.

${ }^{19}$ Oxidation at $\mathrm{Co} / \mathrm{Al}_{2} \mathrm{O}_{3}$ interfaces has been observed in superlattices of these two materials where the Co is polycrystalline and the $\mathrm{Al}_{2} \mathrm{O}_{3}$ is amorphous. See Ch. Morawe and H. Zabel, J. Appl. Phys. 77, 1969 (1995).

${ }^{20}$ W. M. Yim and R. J. Paff, J. Appl. Phys. 45, 1456 (1974).

${ }^{21}$ M. J. Laubitz and T. Matsumura, Can. J. Phys. 51, 1247 (1973).

${ }^{22}$ A. R. Smith, K.-J. Chao, Q. Niu, and C.-K. Shih, Science 273, 226 (1996). 